establish if factors such as gender, serum ACE level and radiographic stage influence sensitivity thresholds.

Methods Twenty-four patients with sarcoidosis (mean (SEM) age 49 (2) years, $63 \%$ female) were recruited from a specialist clinic and underwent assessment of CRS by a single-breath inhalation capsaicin cough challenge test to determine the concentration causing 5 or more coughs $\left(\mathrm{C}_{5}\right)$. Anthropometric data, spirometry, serum ACE levels and radiographic stage were recorded. The effects of gender, age, ethnicity, radiographic stage and serum ACE levels on cough reflex sensitivity were investigated. CRS data of 134 healthy subjects from a previous study were used for comparison (Prudon B et al, Chest 2005;127:550).

Results CRS was heightened in patients with sarcoidosis compared to healthy subjects (geometric mean (logSD) $C_{5} 13.5$ (0.5) vs 158.5 (0.6) $\mu \mathrm{mol} / 1, \mathrm{p}<0.001)$. Female patients had a more sensitive cough reflex compared to males (geometric mean (logSD) $\mathrm{C}_{5} 8.1$ (0.5) vs $31.8(0.5) \mu \mathrm{mol} / \mathrm{l}, \mathrm{p}=0.007)$. Seven patients did not complain of cough; there was no difference in CRS compared to patients who reported cough $(p=0.68)$. There was no difference in CRS between patients of Afro-Caribbean origin compared to non-Afro-Caribbean patients (geometric mean (logSD) $C_{5} 10.1$ (0.5) vs 24.3 (0.6) $\mu \mathrm{mol} / \mathrm{l}$, $p=0.09$ ). Serum ACE levels correlated significantly with $\log C_{5}$ $(\mathrm{r}=0.74, \mathrm{p}<0.001)$, with lower ACE levels being associated with a more sensitive cough reflex. There was no relationship between $\log C_{5}$ and age $(r=-0.40, p=0.054)$ or radiographic stage $(p=0.83)$.

Conclusions Patients with sarcoidosis have a heightened CRS. This was increased to a greater degree in females, but age or radiographic stage had no effect. We report for the first time a link between serum ACE levels and cough reflex sensitivity, and hypothesise that low concentrations of serum ACE lead to increased airway tussigenic mediators such as bradykinin. Further studies should investigate whether cough receptors such as TRPV1 are upregulated in sarcoidosis.

\section{S137 THE NATURAL HISTORY OF IPF IN PATIENTS ELIGIBLE FOR CLINICAL TRIALS VS PATIENTS NOT ELIGIBLE}

\section{doi:10.1136/thoraxjnl-2011-201054b.137}

${ }^{1} \mathrm{P}$ Macfarlane, ${ }^{1} \mathrm{Z}$ H Hoo, ${ }^{1} \mathrm{R}$ L S Hammersley, ${ }^{1} \mathrm{C}$ M McErlean, ${ }^{1} \mathrm{~S}$ Anpalakhan, ${ }^{1} \mathrm{G}$ A Stewart, ${ }^{1} \mathrm{~W}$ A Wallace, ${ }^{1} \mathrm{~J}$ T Murchison, ${ }^{2} \mathrm{~A} J$ Simpson, ${ }^{1} \mathrm{~N}$ Hirani. ${ }^{1}$ University of Edinburgh, Edinburgh, UK; ${ }^{2}$ Newcastle University, Newcastle, UK

Recruitment to clinical trials is a key objective in the management of IPF. For phase 3 trials, the inclusion and exclusion criteria are stringent. It is not known if the natural history of IPF in patients eligible for clinical trials differs from that in non-eligible patients.

Aims To determine the natural history of IPF in patients eligible for phase 3 trials vs those not eligible

Methods Since $1 / 1 / 2002$, all patients with IPF presenting to the Edinburgh Royal Infirmary lung fibrosis clinic have been recruited

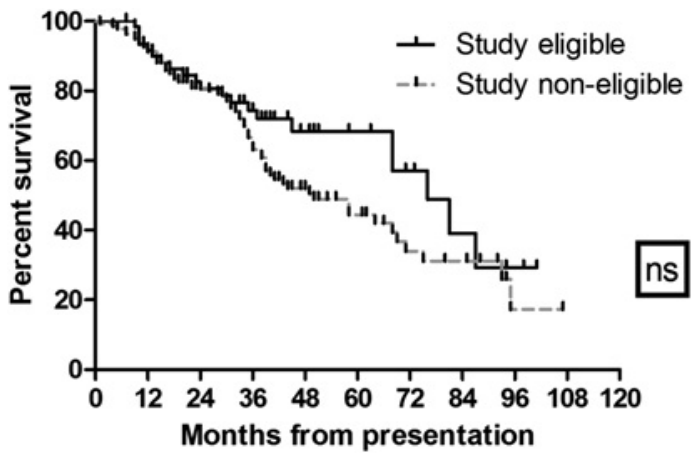

Abstract S137 Figure 1 IPF survival. prospectively to a database. The diagnosis of IPF was made by multi-disciplinary consensus after integration of clinical, HRCT and pathological data, based on ATS/ERS criteria. Management and follow-up was by standardised protocol. IPF-directed therapy, including corticosteroids, azathioprine and anti-oxidants, was considered only in advanced disease, acute exacerbation or in those who exhibited pre-specified fall in lung function. Patients were grouped into those eligible for phase 3 clinical trials and those ineligible, based on the major inclusion/exclusion criteria used in a recently published study (CAPACITY, Lancet 2011 377;1760-1769). Results Of 199 consecutively presenting patients with IPF, 61 (31\%) were eligible for a phase 3 trial. The proportion of males in the eligible and ineligible groups was similar, but eligible patients were younger (68 vs 74 yrs, $\mathrm{p}<0.0001$ ), comprised fewer individuals with $>20$ pack/year smoking history ( $50 \%$ vs $65 \%, p=0.057$ ), had lower $\%$ predicted VC (82.6 vs $95.8 \mathrm{p}=0.0003$ ) and higher \% predicted TLCO (56.6 vs 51.9, p=0.07). Eligible patients had less \% emphysema on HRCT scoring compared to non-eligible patients $(0.74 \%$ vs $6 \% \mathrm{p}<0.0001)$. The 3yr-survival of eligible and ineligible patients were not significantly different (Abstract S137 figure 174\% vs 63\%, $\mathrm{p}=0.3$ ). Event-free survival, defined as time to death or $=10 \%$ fall in VC or $=15 \%$ fall in TLCO or acute exacerbation of IPF or hospital admission with respiratory illness, was not significantly different between eligible and ineligible groups, such that in both groups $40 \%$ and $60 \%$ experienced a progression-defining event by 12 -and 24 months respectively.

Conclusions Trial ineligible patients are demographically and phenotypically different from eligible patients, but have identical mortality and progression-free survival. These data have important implications for translation of trial data to clinical practice and for IPF trial design.

\section{S138 TREATING IDIOPATHIC PULMONARY FIBROSIS WITH THE ADDITION OF CO-TRIMOXAZOLE}

doi:10.1136/thoraxjnl-2011-201054b.138

${ }^{1} \mathrm{~L}$ Shulgina, ${ }^{2} \mathrm{~A}$ Cahn, ${ }^{3} \mathrm{E}$ Chilvers, ${ }^{3} \mathrm{H}$ Parfrey, ${ }^{1} \mathrm{~A}$ Clark, ${ }^{1} \mathrm{E}$ Wilson, ${ }^{4} \mathrm{O}$ Twentyman, ${ }^{5} \mathrm{~T}$ Davison, ${ }^{4} \mathrm{~J}$ Curtin, ${ }^{1} \mathrm{~A}$ Wilson. ${ }^{1}$ University of East Anglia, Norwich, UK; ${ }^{2}$ Bedford Hospital NHS Trust, Bedford, UK; ${ }^{3}$ CUHNHSFT and Papworth Hospitals, Cambridge, UK; ${ }^{4}$ Norfolk and Norwich University Hospital NHS Foundation Trust, Norwich, UK; ${ }^{5}$ Southend University Hospital NHS Foundation Trust, Westcliff-on-Sea, UK

Background Idiopathic pulmonary fibrosis is a fatal condition with limited treatment options; however in a previous small study cotrimoxazole has been shown to be beneficial.

Methods In a double-blind, multi-centre study, 181 patients with usual interstitial pneumonia $(n=166)$ or fibrotic non-specific interstitial pneumonia $(n=15)$ were randomised to receive co-trimoxazole $960 \mathrm{mg}$ twice daily or placebo for 12 months in addition to their usual care. Measurements were made of forced vital capacity (FVC), total lung capacity, total lung diffusing capacity of carbon monoxide, Medical Research Council dyspnoea score, St George's Respiratory Questionnaire and quality adjusted life years (OALYs). All cause mortality, costs and adverse events were recorded.

Results Co-trimoxazole had no effect on FVC or other measures of lung function. However in the per-protocol analysis, co-trimoxazole resulted in a significant reduction in mortality (HR of $0.2(0.06$, $0.78)$ ), significant improvements in the symptom domain of $\mathrm{St}$ George's Respiratory Questionnaire (mean difference -5.30 $(-11.99,1.40)$ units) and OALYs gained (mean difference $0.12(0.01$, 0.22 ) OALYs), and a reduction in the percentage of patients requiring an increase in oxygen therapy (OR $0.05(0.00,0.61)$ ) compared to placebo. Furthermore, the use of co-trimoxazole reduced respiratory tract infections. The incremental cost per OALY gained was $£ 21391$ (52.74\% probability of being below $£ 30000$; intention to treat analysis, UK societal perspective). 